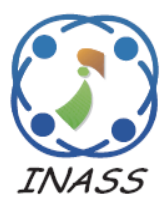

\title{
Spatial Mutual Relationship Based Retinal Image Contrast Enhancement for Efficient Diagnosis of Diabetic Retinopathy
}

\author{
Yarragudi Madhu Sudhana Reddy ${ }^{1 *} \quad$ Ramaswami Sachidanandan Ernest Ravindran ${ }^{1}$ \\ Kakarla Hari Kishore ${ }^{1}$ \\ ${ }^{1}$ Department of Electronics and Communication Engineering, \\ Koneru Lakshmaiah Education Foundation, Vaddeswaram, Guntur, Andhra Pradesh, 522502, India \\ * Corresponding author's Email: ymsr1016@gmail.com
}

\begin{abstract}
Retinal fundus images play an important role in the diagnosis of Diabetic Retinopathy. The detailed information of retinal images like Retinal Vessels, Exudates and microaneurysms may be in low contrast and retinal image enhancement helps in the accurate diagnosis of retinal images related diseases. This paper proposes a novel contrast enhancement method to make the low-contrast retinal images more qualitative such that the detailed information will be clearer. For this purpose, the proposed mechanism considers the spatial mutual relationships between the gray-levels of image and makes the gray-levels in the output image not only linear to the gray-levels of input image but also related to the neighboring gray-levels. The proposed approach enhances the image both in global and local fashion. Spatial mutual entropy based contrast enhancement is accomplished for global contrast enhancement and Greedy contrast enhancement is accomplished for local contrast enhancement. Extensive simulations carried out over various low-contrast retinal images of High Resolution Fundus (HRF) image database shows the outstanding performance of proposed approach. And, different from some other fundus image enhancement methods, the proposed method can directly enhance color images.
\end{abstract}

Keywords: Diabetic retinopathy, Fundus images, Contrast enhancement, Entropy, HRF dataset, CII, LIF.

\section{Introduction}

In the recent years, there has been a gradual increase in the number of diabetic patients and it is approximately 65,000 million persons in India [1]. Among the Diabetesrelated eye disease, Diabetic Retinopathy (DR) is the most chronic disease which affects nearly one out of every ten persons with diabetes, according to point prevalence estimates [2]. DR is one of the most important reasons which make the key cause of vision loss, especially in middle-aged people. India has the highest number of diabetics in world with DR steering it to the 6th biggest cause of vision impairment in the country. In addition, this disease will experience a high growth in the future due to diabetes incidence increase and ageing population in the current society. The early diagnosis allows, through appropriate treatment, to reduce costs generated when they are in advanced states and may become chronic. This fact justifies screening campaigns However, a screening campaign requires a heavy workload for trained experts in the analysis of anomalous patterns of each disease which, added to the at-risk population increase, makes these campaigns economically infeasible. Therefore, the need for automatic screening systems is highlighted. The working process of an automatic screening system follows an organized procedure in which the system tries to learn the characteristics of disease through the retinal images and then the testing is carried out. The automatic system requires a clear and distortion free retinal image to learn its characteristics. Hence there is a need to develop an approach which produces a clear and distortion free image even though the image captured through camera is not clear and contains distortions. As a standard image 
modality, fundus camera is usually used to acquire retinal images, showing structures like optic disc, retinal vessels and several others. The changes detected in these structures indicate the pathological condition associated with diabetic retinopathy. Therefore, the analysis of retinal images is a useful and helpful diagnostic tool.

Due to imaging configuration, retinal images acquired via fundus camera often have low intensity contrast. Some of the major contributing factors may include, curved surface of the retina, degree of dilation, unexpected movements of the patient's eye and other diseases such as cataract [3]. Low contrast in non-uniform illumination is often caused by the first three factors. On the other hand, blurry retina is commonly found due to cataract, which prevents the light from reaching the retina. In general, different types of pathologies may emerge in a retina simultaneously, and a poor quality retinal image may cause hindrance to their clear identification. The analysis over a blurred or low contrast image results in an inaccurate diagnosis and hence there is a necessity to develop an effective image preprocessing approach by which the low contrast image can be boosted to a high contrast image.Generally, contrast enhancement algorithm tries to improve the contrast of image by which the detailed analysis is possible through the clear observation of retinal image features like optic disc, retinal vasculature, and exudates etc. There are so many approaches proposed in earlier to perform contrast enhancement in the retinal images with an aim to increase the detection accuracy in the detection DR [4, 5]. Generally, contrast enhancement methodsare classified as global contrast enhancement (GCE) and local contrast enhancement (LCE). In GCE, the enhancement isbased on the equalization of the histogram of the entireimage. So using only global information is often not enoughto achieve an efficient enhancement in the contrast. To overcome this problem, LCE is applied that considers local window for each individual pixel and computes new intensity values based on local window. If there is a small intensity range, the noise in that region gets more enhanced. Further, some more approaches are proposed by combining the GCE and LCE, named as hybrid contrast enhancement (HCE) approaches. In HCE, initially the image is processed for global contrast enhancement and then for local contrast enhancements by transforming the output of GCE using Discrete cosine transform (DCT), Short Time Fourier Transform (STFT) etc. Recently a hybrid contrast enhancement approach is proposed in [6] to achieve the contrast enhanced image both in global and local fashion. Spatial Entropy Based Contrast Enhancement in DCT (SECEDCT) performs the global contrast enhancement by mapping each input gray-level to an output gray-level through a weight vector measured from the spatial entropy of graylevels. A slight improvement is observed in the output image due to the weight evaluation, however [7] didn't considered the spatial relationships of gray-levels, hence almost all the output gray-levels are linearly related to the input gray-levels and not kept any relation with neighbor gray-levels.

This paper proposes a new hybrid contrast enhancement approach to increase the contrast for the Low contrast retinal images. This method considers the spatial mutual relationship between the gray-levels of input image to find respective optimal gray-level in the output image. A weight between two gray-levels is computed according to their dependence and spatial spread over the image which is quantified as Mutual Relation (MR). The output of the MR is used for mapping the input gray-levels to output. MR quantifies proximity and spatial distribution of two gray-levels over image which is used by ranking mechanism to allocate larger gaps between consecutive gray-levels with high MR values which results in higher perceived contrast on the output image. Further the output is processed for local contrast enhancement by applying greedy mechanism over the global contrast enhanced image.

Rest of the paper is organized as follows; section 2 illustrates the details of literature review. The complete detail of proposed approach is illustrated in section 3. The details of Spatial Entropy based contrast enhancement and spatial mutual entropy based contrast enhancement are illustrated in this section. Section 4 reveals the details of performance evaluation and finally section 5 concludes the paper.

\section{Literature survey}

A clear observation is required for an accurate detection of DR in the retinal images. To achieve this objective, the input retinal image needs to be reprocessed in which the low contrast images are getting enhanced into high contrast images. Thus, a lot of research has been carried out considering the contrast enhancement as a main objective in automatic detection of DR. Global Histogram equalization (GHE) $[8,9]$ and histogram matching are the most common contrast enhancement methods that are applied for DR detection in retinal images. GHE uses an input-to-output mapping derived from matching of the cumulative distribution function (CDF) of input image histogram to $\mathrm{CDF}$ of uniform distribution. Although 
GHE utilizes the available dynamic range of the image, it tends to over-enhance the image if there are large peaks in the histogram, resulting in a harsh and noisy appearance of the enhanced image. Some more approaches are proposed based on the histogram equalization like weighted threshold histogram equalization [10], histogram modification framework [11], and adaptive gamma correction with weighting distribution [12]. However, processing low contrast image through the histograms results in an inadequate contrast enhancement.

Wang et.al, [10] Provides satisfactory enhancement with the carefully selected default parameter setting. Arici et al, [11] minimizes a parameterized cost function to compute a target histogram. The cost function is composed of penalty terms of minimum histogram deviation from the original and uniform histograms, and histogram smoothness. Similar to [10], adaptive gamma correction with weighting distribution [12] modifies the input histogram by weighting distribution and enhances image automatically using gamma correction, however, the algorithm may result in loss of details on bright regions of image when there are high peaks in the input histogram. To overcome this drawback, contrast limited adaptive histogram equalization (CLAHE) is proposed [14]. It is a method for retinal vessel enhancement AHE showed amplifying noise in some of the homogenous regions, thus to eliminate this problem CLAHE was proposed. Based on the CLAHE mechanism, an improved version, Rayleigh transformation in contrast-limited adaptive histogram equalization (RCLAHE) was proposed by J. tasuttisak et al, [16] to preserve color information of the retinal images. However in the contrast enhancement through CLAHE methods needs an optimal parameter setting to perform appropriate contrast enhancement at individual grey levels which results in the less robustness. i.e., a parameter fixed for one type/level of images is not able to perform better achievement in the contrast levels for another type of images.

In [20], an investigation of three enhancement methods namely, Histogram Equalization (HE), Contrast Limited Adaptive Histogram Equalization (CLAHE) and Mahalanobis Distance (MD), were conducted on the digital fundus images and the results are qualitatively presented using histogram representation and image product quality. But the CLAHE method [15, 19-20] may produce artificial boundaries at the region which has an abrupt change in the gray levels and also not able to preserve the mean brightness of retina images which was very important for the diagnosis of retinal fundus images.
To preserve the mean brightness of retinal images, many brightness-preserving techniques were introduced earlier. Dynamic histogram equalization (DHE) [17] is one of them, where mean image brightness is more or less varied due to histogram peak remapping. In DHE, the image histogram partitioned using the position of local minima. This method is not good enough for preserving the mean brightness due to the fact that remapping of histogram peaks caused mean brightness to also vary.

Ibrahim and Kong [17] proposed the brightnesspreserving DHE (BP-DHE) to avoid the peak remapping of histogram. This method applies the crisp histograms to enhance the contrast of the image but this technique does not take any consideration of inexact gray values. So, mean brightness significantly changes. In [18], Vessel Enhancement was done via multi-dictionary and sparse coding. In this method, two corresponding dictionaries are generated one is the Representation Dictionary (RD) and the other one is the Enhanced Dictionary (ED). The patches in RD and ED are selected through the information images to optimize the multi-dictionary. U. Qidwai [21] proposed a retinal image enhancement method based on the blind deconvolution approach using maximum likelihood estimation. And this deconvolution approach showed a promising result, which may be used for clinical application. Bai et al. [22] proposed an image enhancement method based on multiscale top-hat transformation. In this method, the image details were well enhanced, but the contrast is not improved effectively.

Rampal et al. [23] used a complex diffusionbased shock filter for retinal image smoothing and contrast enhancement. And this method outperformed other methods on the DIARETDB1 database. Liao et al. [24] used a hybrid model of multiscale top-hat transformation and histogram fitting stretching to enhance retinal fundus image, which could enhance the contrast of the retinal image effectively and highlight the retinal vessels well. However the retinal image contrast enhancement through these approaches leads to artificial boundaries, abrupt changes in color levels, and the loss of image detail. In order to avoid these side effects, a new retinal fundus image enhancement method is proposed in [25] through Normalized convolution and noise removing. The noise in the retinal image is filtered through Partial Differential Equations and Relaxed Median Filter. 


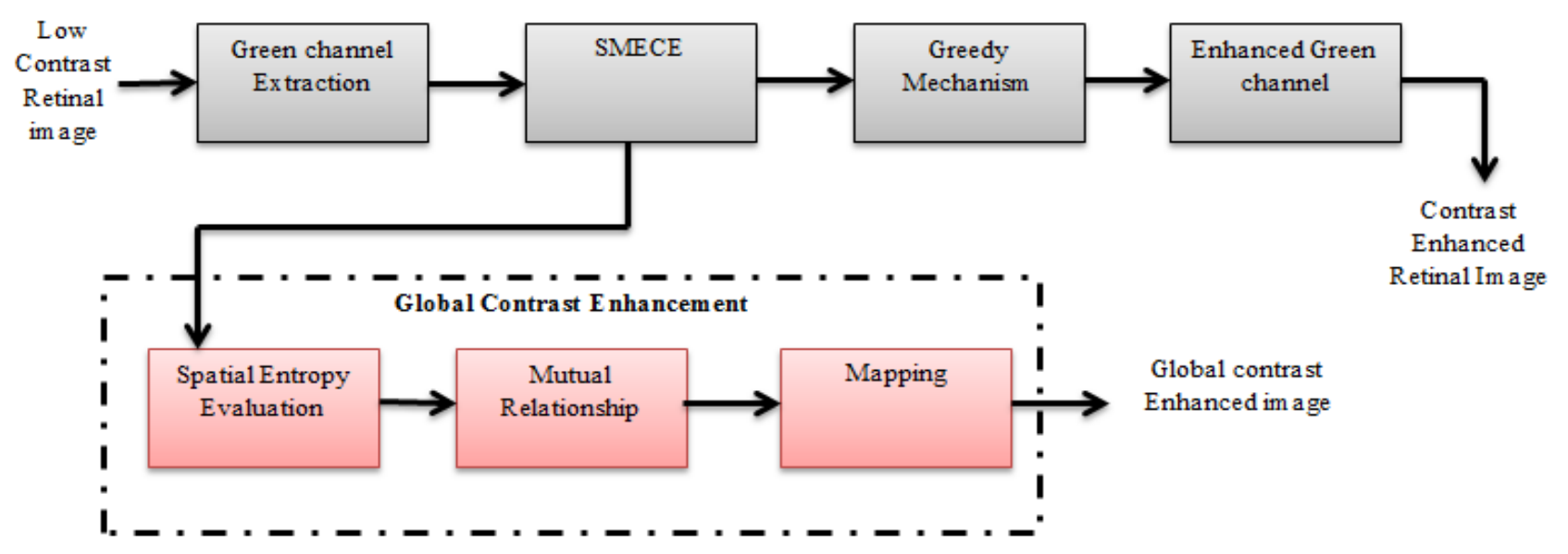

Figure. 1 Overall block diagram of proposed approach

\section{Proposed approach}

In this section, the complete details of proposed approach are outlined briefly. The proposed considers the spatial mutual relations between the gray-levels for contrast enhancement whereas the conventional approach didn't considered these spatial dependencies by which the gray-levels in the contrast enhanced image don't have any relationship with neighboring pixels which makes the entire process more complex and not clear. Overall block diagram of proposed mechanism is shown in Fig. 1, above.

Fig. 1 shows the overall block diagram of proposed mechanism. The proposed approach tends to achieve the contrast of an image both globally and locally. Hence the proposed approach is accomplishes the global contrast enhancement and further the local contrast enhancement. The complete details or proposed approach is outlined as follows;

Consider an input image ' $I$ ' with size $H \times W$ which has gray-levels range of $\left[I_{d}, I_{u}\right]$ and the enhanced image ' $O$ ' of range $\left[O_{d}, O_{u}\right]$, which has better visual quality than the input image ' $I$ '. Mathematically these input and output images are represented as,

$I=\{I(m, n) \mid 0 \leq m \leq M-1,0 \leq n \leq N-1\}$

$O=\{O(m, n) \mid 0 \leq m \leq M-1,0 \leq n \leq N-1\}$

Here the range of input and output images such as $I(m, n) \in\left[I_{d}, I_{u}\right]$ and $O(m, n) \in\left[O_{d}, O_{u}\right]$ varies from 0 to 255 for an 8-bit image. This range is ideal, i.e., 0-255 only if the enhancement process utilizes the entire dynamic gray-level range. In such case, the lower limit $O_{d}=0$ and the upper limit $O_{u}=$ 255.

\subsection{Spatial entropy based contrast enhancement (SECE) [7]}

Let the input image $I$ has $K$ distinct gray levels and are sorted in an ascending order like $\left\{I_{1}, I_{2}, \ldots, I_{K}\right\}$. An input image $\mathrm{I}$ is divided into $\mathrm{H}^{*} \mathrm{~W}$ spatial grids. The 2D spatial histogram of a graylevel $I_{k}$ on the spatial grid of I is computed as,

$$
h_{k}=\left\{h_{k}(h, w) \mid 1 \leq h \leq H, 1 \leq w \leq W\right\}
$$

Where $h_{k}(h, w)$ defines the number of occurrences of gray-level $I_{k}$ in the spatial grid located in the image region of $\left[\left((h-1) \times \frac{M}{H}, h \times \frac{M}{H}\right) \times\right.$ $\left.\left((w-1) \times \frac{N}{W}, w \times \frac{N}{W}\right)\right]$. The total number of grids on the 2D histogram is HW which was estimated dynamically using the aspect ratio, $r=\frac{M}{N}=\frac{H}{W}$.

$$
W=\left\lfloor\left(\frac{K}{r}\right)^{1 / 2}\right\rfloor \text { and } H=\left\lfloor(K r)^{1 / 2}\right\rfloor
$$

Where the operator [.] makes the argument round off to its nearest neighbor value.

In the every grid, the distribution of a gray-level $I_{k}$ can be measured trough the spatial entropy and the spatial entropy $S_{k}$ for a gray-level $I_{k}$ is obtained according to

$$
S_{k}=-\sum_{h=1}^{H} \sum_{w=1}^{W} h_{k}(h, w) \log _{2} h_{k}(h, w)
$$

Further, the obtained spatial distribution has to evaluate with respect to the distributions of the other gray-levels to know the importance and it is measured through a discrete function $f_{k}$ as,

$$
f_{k}=S_{k} / \sum_{l=1, l \neq k}^{K} S_{l}
$$


Further the obtained discrete function $f_{k}$ is normalized as,

$$
\hat{f}_{k}=f_{k} / \sum_{l=1}^{K} f_{l}
$$

And based on the obtained normalized discrete function $\hat{f}_{k}$, a cumulative distribution function $F_{k}$, of a gray-level $I_{k}$ is defined as,

$$
F_{k}=\sum_{l=1,}^{k} f_{l}
$$

Finally the gray-level of an output image $\mathrm{O}$ is obtained through the following mapping function.

$$
O_{k}=\left\lfloor F_{k}\left(O_{u}-O_{d}\right)+O_{d}\right\rfloor
$$

The final output $\mathrm{O}$ denotes the contrast enhanced image. Further the performance $f$ this mechanism is measured through the performance metrics like expected measure of enhancement by gradient (EMEG) and gradient magnitude similarity deviation.

\subsection{Spatial mutual entropy based global contrast enhancement (SMECE)}

Though the conventional approach achieved an increased contrast in the output image, this method didn't consider the spatial relationships between the gray levels, hence, most of the time the output graylevels are simply a linear mapping of input graylevels, i.e., a particular gray-level in the output image is just linearly related to the input gray-level and don't have any mutual relationship with the other gray-levels. In order to address this issue, this work proposes a new contrast enhancement mechanism by considering the spatial mutual relations between the gray-levels. To obtain a normalized 2D spatial histogram, the 2D histogram entries are further normalized as

$$
h_{k}(h, w)=h_{k}(h, w) / H W
$$

Such that

$$
\sum_{k=1}^{K} \sum_{h=1}^{H} \sum_{w=1}^{W} h_{k}(h, w)=1
$$

Here the spatial mutual entropy is measured by evaluating the joint 2D spatial histograms for a given two gray-levels $I_{k}$ and $I_{l}$ on the spatial grid located on the image region of $\left[\left(h \times \frac{M}{H},(h+1) \times\right.\right.$ $\left.\left.\frac{M}{H}\right) \times\left(w \times \frac{N}{W},(w+1) \times \frac{N}{W}\right)\right]$, as

$$
h_{k, l}(h, w)=\min \left(h_{k}(h, w), h_{l}(h, w)\right)
$$

The above expression gives a new evaluation procedure to measure the spatial relationships between two gray-levels $I_{k}$ and $I_{l}$. Further to obtain the spatial dependencies of gray-levels and their spatial spread over the image domain, a new metric called Mutual Relation (MR) is derived here and formulated as,

$$
M R_{k, l}=\sum_{h=0}^{H-1} \sum_{w=0}^{W-1} h_{k, l}(h, w)\left(\frac{h_{k, l}(h, w)}{h_{k}(h, w) h_{l}(h, w)}\right)
$$

Where $M R_{k, l}$ is the mutual relation between two gray-levels $I_{k}$ and $I_{l}$. The above expression gives a spatial relationship between the gray-levels $I_{k}$ and $I_{l}$. The Mutual Relation is high when the gray-levels $I_{k}$ and $I_{l}$ occur jointly on close spatial regions and spread over the image domain. Thus, this method can allocate higher gap between the gray-levels $O_{k}$ and $O_{l}$ such that the obtained contrast will be high.

After measuring he mutual relations for every gray-level with respect to all other gray-levels, one rank is assigned to that relation based on the closeness and finally the output gray-level $O_{k}$ is obtained through the following mapping function,

$$
O_{k}=\left\lfloor O_{k-1}+\Delta_{k-1, k}\left(O_{u}-O_{d}\right)\right\rfloor
$$

Where

$$
\Delta_{k-1, k}=\frac{r(k-1)+r(k)}{2}
$$

The term $\Delta_{k-1, k}\left(O_{u}-O_{d}\right)$ in Eq. (13) is the gap between consecutive output gray-levels $O_{k-1}$ and $O_{k}$. This gap is determined according to joint contribution of consecutive gray-levels which is defined as average of their ranks. The mapping function always guarantees that the minimum and maximum values of output gray-levels are the minimum and maximum of allowed dynamic range. Thus the proposed approach efficiently utilizes the allowed dynamic range and produces an effective contrast enhanced image.

\subsection{Local contrast enhancement}

According to the [26] the perceived contrast of an image is directly related to the local gradient of image. Hence to achieve an optimal local contrast in the local region, the gradient of images needs to optimized subjecting to strict constraints that prevent both over/under-saturation and unbounded 
enhancement of the gray values. Thus, we propose to maximize the objective function as

$$
f(\omega)=\frac{1}{4|\omega|} \sum_{m \in \omega} \sum_{n \in N_{4}(m)} \frac{O(m)-O(q)}{I(m)-I(n)}
$$

Subjecting to the constraint of

$$
\begin{gathered}
1 \leq \frac{O(m)-O(q)}{I(m)-I(n)} \leq(1+\gamma) \\
L \leq O(m) \leq U
\end{gathered}
$$

Where $I(\mathrm{~m})$ and $O(\mathrm{~m})$ are the gray values at the pixel value $m$ of the input and output images respectively, $\omega$ denotes set of pixels that makes up the image, $|\omega|$ denotes the cardinality of $\omega$, $N_{4}(m)$ denotes the set of four neighbors of $\mathrm{m}, \gamma$ is a single parameter that can only controls the level of contrast can be achieved, $\mathrm{L}$ and $\mathrm{U}$ are the lower and upper bounds of gray-levels $(\mathrm{L}=0$ and $\mathrm{U}=255)$. Maximizing our objective function results in pronouncement of the local gradient around a pixel in the input image to the maximum possible degree. The expression shown in Eq. (16) assures the contrast enhancement of an image through its gradients. The lower bound assures the preservation of signs of gradient and prevents the image form shrinking and the upper bound assures the bounded enhancement of the image through the controlling parameter $\gamma$. Further the expression shown in Eq. (17) assures the non-saturated values of output image.

To optimize the problem formulated in Eqs. (16) and (17), a greedy mechanism [27] is accomplished in an iterative fashion. Let us consider an image $I$ as a height-width sampled at grid points of $h \times w$ uniform grid. This set of samples represents $\omega$ for an $h \times w$ image. Thus the width at every pixel $m \in$ $\omega, I(m)$ is within $\mathrm{L}$ and $\mathrm{U}$. In every iteration, this approach considers a new variable $t, L \leq t \leq U$. Next a matrix $R$ of size $h \times w$ is generated based on the $t$ value, as

$$
R(i, j)= \begin{cases}1, & \text { if } I(i, j)>t \\ 0, & \text { if } I(i, j) \leq t\end{cases}
$$

Next, the non-zero components in the $\mathrm{R}$ matrix are traced and labelled uniquely as $e_{t}^{i}$, eminence, where $t$ denotes the threshold value defined to declare the eminences and $i$ is the component number. Further the obtained eminences are pushed up such that no pixel belonging to the eminence has the gradient around it enhanced by a factor more than $(1+\gamma)$ and is pushed up beyond $\mathrm{U}$.
In this fashion this approach sweeps the threshold value from $\mathrm{L}$ to $\mathrm{U}$ and in every iteration, the eminences are scaled greedily with proper constraints. For low values of $t$, the enhancement will not be greater than $(1+\gamma)$, because there is a chance of eminences to achieve the peak values which are close to $\mathrm{U}$. As the threshold value $t$ increases, the larger connected components are divided further into smaller eminences and can be enhanced more than before. This establishes the upward sweep in the proposed approach which enhances only the eminences of $I$ and the image thus obtained can be represented as $I_{l}$. The further enhancement is carried out through the valley also. So the second stage of this approach applies the same methodology to the compliment of $I_{1}=U-$ $I_{1}(m)$ to obtain the new output $I_{2}$. Finally the $I_{2}$ is complimented again to obtain the output enhanced image $O=U-I_{2}(m)$.

Totally the proposed local contrast enhancement mechanism performs U-L sweeps to generate $I_{1}$ and $I_{2}$. At every sweep, the connected components are identified from the $h \times w$ matrix. Thus the timecomplexity of this approach is denoted as $O((U-$ $L)(h w+\log (U-L)))$. Since this approach is completely design in the range of gray-level image, to achieve the contrast enhancement for the color images, the time-complexity becomes the thrice of gray-level image complexity, i.e. $3 \times(O((U-$ $L)(h w+\log (U-L))))$.

\section{Simulation results}

To evaluate the performance or proposed approach, the low contrast images are collected from High Resolution Fundus (HRF) database [28]. This database is prepared by capturing 18 pairs of the same eye 18 human subjects using a Canon CR-1 fundus camera with a field of view of $45^{\circ}$ and different acquisition setting. For each pair, the first image has poor quality and thus the examination had to be repeated. Both images share approximately the same field of view, whereas small shifts were caused by eye movements between the acquisitions. The poor quality images are considered for the performance evaluation and the proposed approach is applied over those images. This poor quality dataset was captured by Jan Odstrcilik [29].

The image enhancement results of the proposed method are color images. Yet, the green channel image is generally used to evaluate image enhancement results. So only the green channel of the enhanced image is used in image evaluation step. The comparative analysis is carried out with 
Table 1. Contrast enhanced images through proposed and conventional approaches

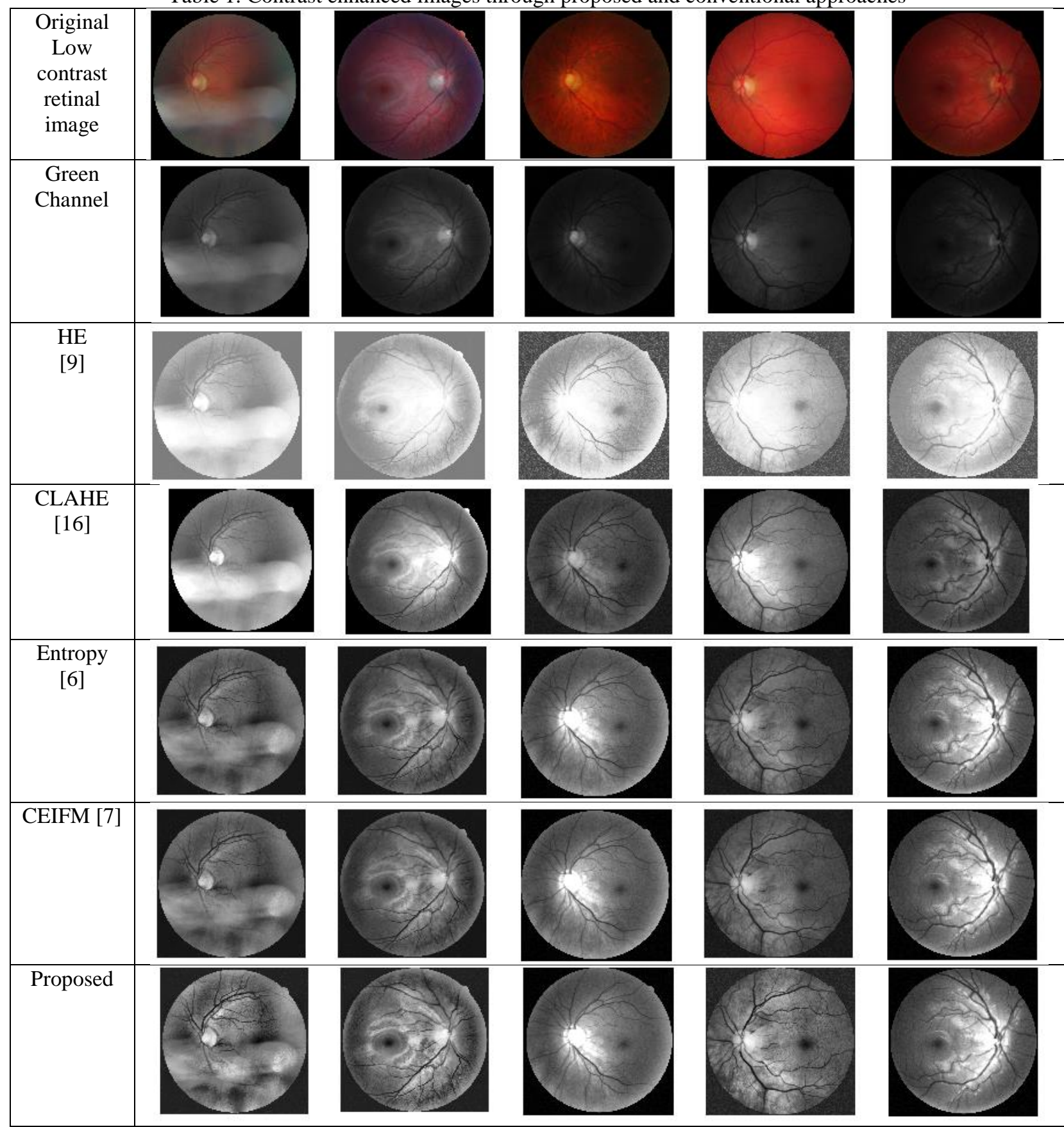

Histogram Equalization, Contrast Limited Adaptive Histogram Equalization [16], Spatial Entropy Based Contrast Enhancement [6] and Contrast Enhancement through Image Formation model (CEIFM) [7]. The obtained results for the HRF dataset are shown in Table 1.

Table 1 shows the contrast enhanced images through the proposed SMECE and the conventional HE, CLAHE, entropy based and CEIFM approaches. From the last row of the table.1, it can be obserevd that the proposed approach obtained better enhancement both in the vessel-regions and nonvessel regions of fundus images. Compared to the conventional HE, CLAHE, Entropy and CEIFM, the proposed approach is observed to achieved the better visual results. The vessel regions are more clear in the enhenced image obtained through the proposed approach compared to the conventional approaches.

Further theperformance of proposed approach is measured with respect to two performance metrics, contrast improvement index (CII) [30] and linear index of fuzziness (LIF) [22]. The CII is measured as,

$$
C I I=\frac{C_{e n}}{C}
$$


Table 2. Comparison of CII and LIF for different methods for the results shown in Table 1

\begin{tabular}{|l|c|c|c|c|c|c|c|c|c|c|}
\hline \multirow{2}{*}{ Image } & \multicolumn{2}{|c|}{ HE [9] } & \multicolumn{2}{c|}{ CLAHE [16] } & \multicolumn{2}{c|}{ Entropy [6] } & \multicolumn{2}{c|}{ CEIFM [7] } & \multicolumn{2}{c|}{ Proposed } \\
\cline { 2 - 11 } & CII & LIF & CII & LIF & CII & LIF & CII & LIF & CII & LIF \\
\hline Image 1 & 1.3530 & 0.5526 & 1.3677 & 0.4499 & 2.8506 & 0.3387 & 3.0024 & 0.3012 & 4.9501 & 0.2302 \\
\hline Image 2 & 2.1529 & 0.5220 & 2.1676 & 0.4420 & 3.6505 & 0.2996 & 3.8657 & 0.2887 & 5.7500 & 0.2563 \\
\hline Image 3 & 1.3533 & 0.4578 & 1.3680 & 0.3978 & 2.8509 & 0.2585 & 3.1547 & 0.2374 & 4.6235 & 0.1996 \\
\hline Image 4 & 1.5102 & 0.5220 & 1.6879 & 0.4478 & 3.0078 & 0.3567 & 3.5524 & 0.2978 & 4.6637 & 0.2125 \\
\hline Image 5 & 2.336 & 0.4985 & 2.8674 & 0.4127 & 3.6874 & 0.3385 & 3.9815 & 0.2863 & 5.8897 & 0.2129 \\
\hline
\end{tabular}

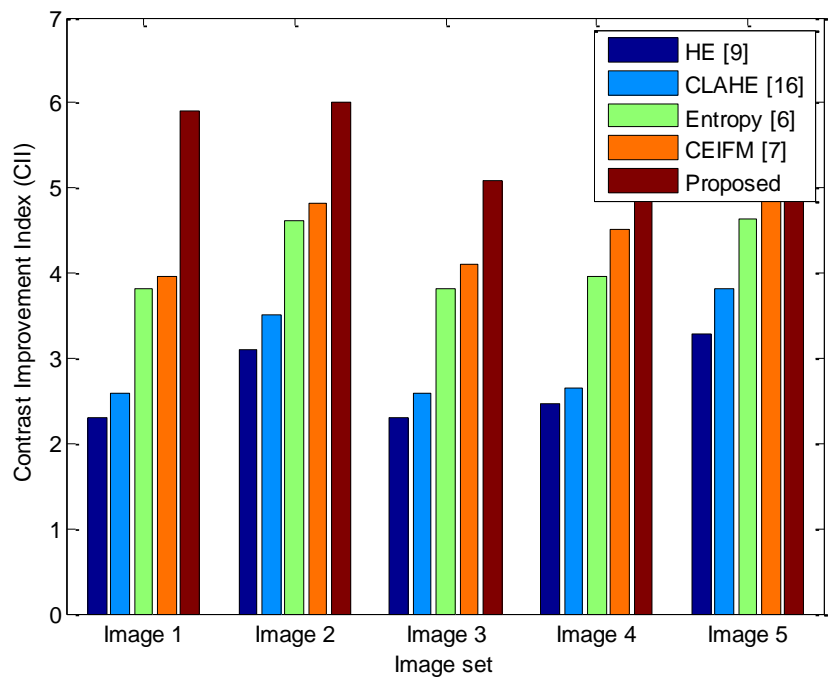

Figure. 2 Comparision of CII

Where $C_{e n}$ and $C$ the contrast values for the retinal vessel in the enhanced image and the original image, respectively. Here the $C_{e n}$ and $C$ are measured without considering the background blackpixels ino acocunt which are outside the pupil. The $\mathrm{C}$ is measured as follows;

$$
C=\left|\frac{f-b}{f+b}\right|
$$

Where $f$ and $b$ are the average gray values of the retinal vessels and the non-vessel regions, respectively. From the above expression, it is clear that the larger $\mathrm{C}$ value indicates the larger difference between the vessel regions and non-vessel regions. In the same way, the $C_{e n}$ is also measured by considering the enhanced image as input. Thus A larger value of CII shows that the retinal vessels are enhanced better. Further the LIF is defined as

$$
L I F=\frac{2}{M * N} \sum_{x=1}^{m} \sum_{y=1}^{n} \min \left\{p_{x y},\left(1-p_{x y}\right)\right\}
$$

Where

$$
p_{x y}=\sin \left[\frac{\pi}{2} \times 1-\left(\frac{f(x, y)}{f_{\max }}\right)\right]
$$

Where $f_{\max }$ is the maximum value of the whole image of size $M \times N$.

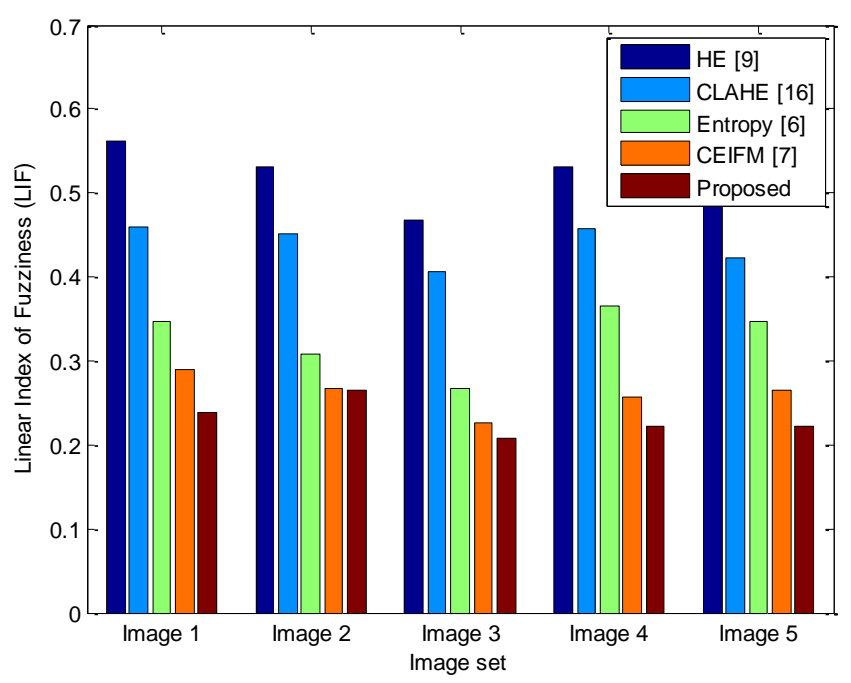

Figure. 3 Comparision of LIF

A smaller value of $L I F$ shows that the whole enhanced image is clearer and has less noise. Thus, a large value of CII and a small value of LIFindicate a good image enhancement method. The obtained CII and LIF values for the above test images are represented in Table 2, above.

Figs. 2 and 3 represent the comparative analysis between the proposed SMECE and the conventional HE, CLAHE, Entropy and CEIFM. Totally the comparison is carried out for five test images and the obtained CII and LIF are observed to be better for proposed approach. Since the proposed approach considered the spatial relationships between the entropy distributions, the enhanced image is not a simple relation of input and output images. A graylevel in the output image is also related to the graylevels of neighboring gray-levels of respective input gray-level. And also the proposed approach follows a greey contrast enhancement for local contrast enhancement of image by which the edge regions are enhanced up to the required limit. Thus the overall image is enhanced through proposed approach is observed to be better compared to the conventional approaches. The performance enhancement achieved through the proposed approach is much better when compared with the conventional approaches due to their individual limitations such as over brightness of HE, no 


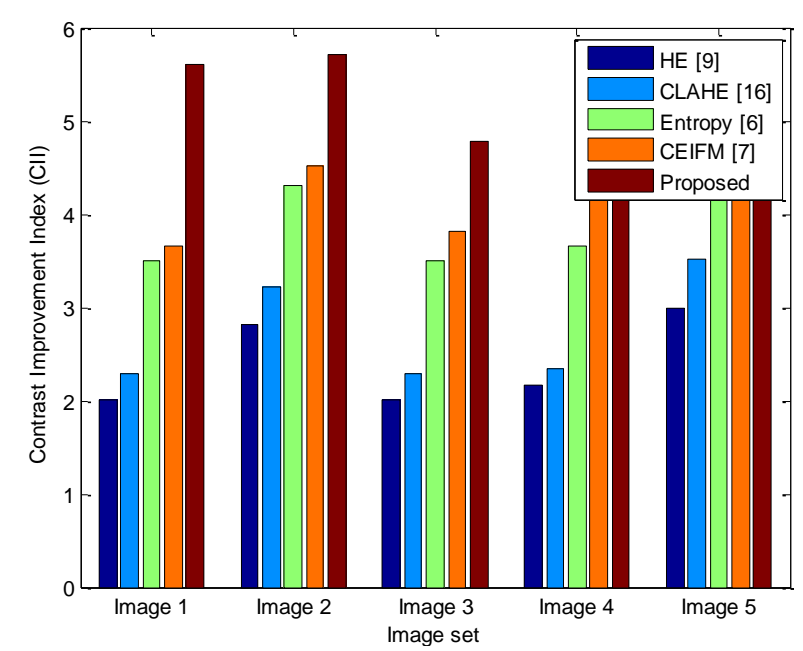

Figure. 4 Comparisonof CII

relation between the enhanced grey levels with neighboring grey levels in CLAHE, Normalization problem with Spatial entropy based contrast enhancement and finally the image formation model constitutes more and more complexity due to various assumption before forming the image with respect to their illumination and reflectance. Figure. 2 and figure. 3 clearly describes the details of performance enhancement through CII and LIF. From both figures, the CII and LIF of proposed SMECE is observed to be more effective when compared to the conventional ones. After enhancing the green channel of a retinal image, the remaining red and blue regions are fused with enhanced green channel to form a contrast enhanced color image. The obtained CII and LIF for color images are shown in Figs. 5 and 6, respectively.

\section{Conclusion and future scope}

In this paper, a novel contrast enhancement approach is proposed to enhance the contrast of lowcontrast retinal images by which the diagnosis accuracy in the automatic Diabetic Retinopathy Detection increases. The proposed approach considered the spatial entropies of gray-levels to perform contrast enhancement. Along with this the proposed approach also considered the spatial mutual entropies by which the gray-level in the output image not only depends on the input graylevel but also on the neighbor gray-levels. This is an ad on technique which booststhe performance in the contrast enhancement. Global contrast enhancement is done through spatial mutual entropies and the local contrast enhancement is done through gray contrast enhancement mechanism. Simulation carried out over various low contrast images and the performance is measured through performance metrics like CII and LIF, revealed the outstanding

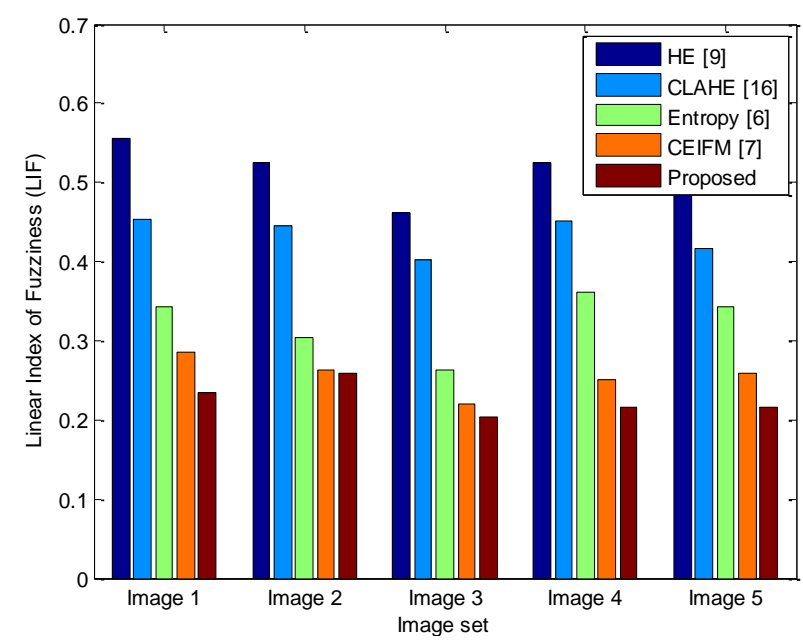

Figure. 5 Compairison LIF

performance of proposed approach. From the simulation results it is observed that the CII and LIF are more efficient for the proposed approach compared to the conventional approaches.

Since the image quality is most important in the automatic DR detection, this paper proposed an efficient contrast enhancement mechanism by which the quality of fundus image increases which results in more accurate diagnosis. This is the first stage in the automatic DR detection. In the future, this work can be extended in many ways. First, to increase the further quality of image, this work extends by developing a new contrast enhancement mechanism based on the image formation model. Second, this work can be extended by developing a novel feature extraction technique by which the features of retinal images such as optic disk and retinal vessel are extracted which are considered for further classification process.

\section{References}

[1] International Diabetes Federation, IDF Diabetes Atlas, $7^{\text {th }}$ ed. Brussels, Belgium: International Diabetes Federation, 2015.

[2] V. Mohan, S.N. Shah, S.R. Joshi, V. Seshiah, B.K Sahay, and S. Banerjee, "Current status of management, control, complications and psychosocial aspects of patients with diabetes in India: Results from the Diab Careindia 2011 study", Indian Journal of Endocrinal Metab., Vol.18, No.3, pp.370-378, 2014.

[3] G.D. Joshi and J. Sivaswamy, "Colour retinal image enhancement based on domain knowledge", In: Proc. of International Conf. on Computer Vision, Graphics \& Image Processing, pp.591-598, 2008.

[4] A.A. Youssif, A.Z. Ghalwash, and A.S. Ghoneim, "Comparative study of contrast 
enhancement and illumination equalization methods for retinal vasculature segmentation", In: Proc. of International Conf. on Biomedical Engineering, pp.1-5, 2006.

[5] A.A. Youssif, A.Z. Ghalwash, and A.S. Ghoneim, "A comparative evaluation of preprocessing methods for automatic detection of retinal anatomy", In: Proc. of International Conf. on Informatics and Systems, pp.24-30, 2007.

[6] T. Celik, "Spatial Entropy-Based Global and Local Image Contrast Enhancement", IEEE Transactions on Image Processing, Vol.23, No. 12, pp.5298-5308, 2014.

[7] L. Xiong, H. Li, and X. Liang, "An Enhancement Method for Color Retinal Images Based on Image Formation Model", Journal of Computer Methods and Programs in Biomedicine, Vol.143, No.3, pp.137-150, 2017.

[8] N.M. Salem and A.K. Nandi, "Novel and adaptive contribution of the red channel in preprocessing of colour fundus images", Journal of Franklin Institute, Vol.344, No.3, pp.2432562007.

[9] M.H.A. Hijazi, F. Coenen, and Y. Zheng, "Retinal image classification using a histogram based approach", In: Proc. of International Conf. on Neural Networks, pp. 1-7, 2010.

[10] Q. Wan and R.K. Ward, "Fast image/video contrast enhancement based on weighted thresholded histogram equalization", IEEE Transactions on Consumer Electronics, Vol. 53, No.2, pp.757-764, 2007.

[11] T. Arici, S. Dikbas, and Y. Altunbasak, "A histogram modification framework and its application for image contrast enhancement", IEEE Transactions on Image processing, Vol. 18, No.9, pp.1921-1935, 2009.

[12] S.C. Huang, F.C. Cheng, and Y.S. Chiu, "Efficient contrast enhancement using adaptive gamma correction with weighting distribution", IEEE Transactions on Image processing, Vol. 22, No.3, pp.1032-1041, 2013.

[13] A. Dehghani, A.M. Hamid, and M. S.Molin, "Optic disc localization in retinal images using histogram matching", EURASIP Journal on Image and Video Processing, Vol.2012, No.19, pp.1-11, 2012.

[14] A.M. Reza, "Realization of the contrast limited adaptive histogram equalization (CLAHE) for real-time image enhancement", Journal of VLSI Signal Processing Systems for Signal, Image and Video Technology, Vol. 38,No. 1, pp. 3544, 2004.
[15] A.W. Setiawan, T.R. Mengko, O.S. Santosa, and A.B. Suksmono, "Color Retinal Image Enhancement using CLAHE", In: Proc. of International Conf. on ICT for Smart Society, pp. 1-3, 2013.

[16] T. Jintasuttisak and S. Intajag, "Color Retinal Image Enhancement by Rayleigh ContrastLimited Adaptive Histogram Equalization", In: Proc. of International Conf. on Control, Automation and Systems, pp. 22-25 2014.

[17] H. Ibrahim and N.S.P. Kong, "Brightness preserving dynamic histogram equalization for image contrast enhancement", IEEE Transactions on Consumer Electronics, Vol.53, No.4, pp.1752-1758, 2007.

[18] B. Chen, Y. Chen, Z. Shao, T. Tong, and L. Luo, "Blood Vessel enhancement via multidictionary and sparse coding: Application to retinal vessel enhancing", Neurocomputing, Vol.200, No.5, pp. 110-117, 2016.

[19] S.K. Shome and S.R. Krishnai, "Enhancement of Diabetic Retinopathy Imagery Using Contrast Limited Adaptive Histogram Equalization", International Journal of Computer Science and Information Technologies, Vol. 2, No.6, pp. 2694-2699, 2011.

[20] H. Rahim, A.S. Ibrahim, W.M. Diyana, and A.Hussain, "Methods to Enhance Digital Fundus Image for Diabetic Retinopathy Detection", In: Proc. of International Colloquium on Signal Processing \& its Applications, pp.221-225, 2014,.

[21] U. Qidwai, "Blind Deconvolution for retinal image enhancement", In: Proc. of the IEEE EMBS Conference on Biomedical Engineering and Sciences, pp. 20-25, 2010.

[22] X.Z. Bai, F.G. Zhou, and B.D. Xue, "Image enhancement using multi scale image features extracted by top-hat transform", Optics and Laser Technology, Vol. 44, No. 2, pp. 328-336, 2012.

[23] H. Rampal, R. K. Kumar, B. Ramanathan, and T. P. Das, "Complex shock filtering applied to retinal image enhancement", In: Proc. of the World Congress on Medical Physics and Biomedical Engineering, pp. 900-903, 2012.

[24] [24] M. Liao, Y.Q. Zhao, X.H. Wang, and P.S. Dai, "Retinal vessel enhancement based on multi-scale top-hat transformation and histogram fitting stretching", Optics and Laser Technology, Vol. 58, No.6, pp. 56-62, 2014.

[25] P. Dai, H. Sheng, J. Zhang, L. Li, J. Wu, and M. Fan, "Retinal Fundus Image Enhancement Using the Normalized Convolution and Noise 
Removing", International Journal of Biomedical Imaging, Vol.2016, pp.1-12, 2016.

[26] R.L.D. Valois and K.K.D. Valois, Spatial Vision, Oxford University Press, Berkeley, 1990.

[27] K. Subr, A. Majumder, and S. Irani, "Greedy algorithm for local contrast enhancement of images", In: Proc. of International Conf. on Image Analysis and processing, pp.171-179, 2005.

[28] A. Budai, R. Bock, A. Maier, J. Hornegger, and G. Michelson, "Robust Vessel Segmentation in Fundus Images", International Journal of Biomedical Imaging, Vol. 2013, No.6, pp.1-11, 2013.

[29] K. Thomas, A. Budai, K. Martin, J. Odstrcilik, G. Michelson, and J. Hornegger, "Automatic No-Reference Quality Assessment for Retinal Fundus Images Using Vessel Segmentation", In: Proc. of International Symposium on Computer-Based Medical Systems, pp.95-101, 2013,

[30] J. Oh and H. Hwang, "Feature enhancement of medical images using morphology-based homomorphic filter and differential evolution algorithm", International Journal of Control, Automation and Systems, Vol. 8, No. 4, pp. 857-861, 2010. 